Grasping the dialogical nature of acculturation

\section{Catarina Rosa}

University of Aveiro, Portugal

\section{Sofia Tavares}

University of Évora, Portugal
(C) The Author(s) 2013

Reprints and permissions: sagepub.co.uk/journalsPermissions.nav DOI: I0.1 I77/I354067XI3478987

\begin{abstract}
In this interesting article, Andreouli (2013). Identity and acculturation: The case of naturalised citizens in Britain. Culture \& Psychology, 19, I-47) presents a dialogical perspective on acculturation. To support this perspective, the author integrates the Dialogical Self Theory and the Social Representations Theory. Drawing on her theoretical explanation, we develop a conceptual review focused on two pairs of constructs social representations/l-positions and polyphasia/polyphonia. Andreouli's empirical study allowed her to operationalize some critiques about the two-dimensional perspective and its strategies on acculturation. Nevertheless, it seems that the author ends up replicating a more conventional and dual way of thinking. Their results give us privileged access to the negotiation of meanings and activation of promoter signs or, in other words, to the dialogical dynamics between I-positions. In this respect, we suggest that the assumption of a more dialogic and semiotic lens could be an interesting further development to this study.
\end{abstract}

\title{
Keywords
}

Acculturation, social representations, I-positions, dialogical dynamics, promoter signs

\section{Introduction}

The postmodern movements introduced a major change in the way we conceptualize and investigate, allowing us to integrate the dynamic characteristics of the contemporary world. Individuals have now more freedom and more options, which represent, on the one hand, an increase of possibilities but, on the other, a risk of

\section{Corresponding author:}

Catarina Rosa, University of Aveiro, Campus Universitário de Santiago, Aveiro 3810-193, Portugal.

Email: catarina.rosa@gmail.com 